\title{
In Vivo Scanning Laser Confocal Microscopy of Conjunctival Goblet Cells in Medically-controlled Glaucoma
}

\author{
SILVIO DI STASO ${ }^{1}$, LUCA AGNIFILI $^{2}$, MARCO CIANCAGLINI $^{1}$, \\ GIANLUCA MURANO $^{1}$, ENRICO BORRELLI ${ }^{2}$ and LEONARDO MASTROPASQUA ${ }^{2}$ \\ ${ }^{1}$ Department of Life, Health and Environmental Sciences, University of L'Aquila, \\ Ophthalmology Clinic, L'Aquila, Italy; \\ ${ }^{2}$ Ophthalmology Clinic, Department of Medicine and Science of Ageing, \\ University G. D'Annunzio Chieti Pescara, Chieti, Italy
}

\begin{abstract}
Aim: The aim of this study was to evaluate the goblet cell density (GCD) of conjunctiva in medicallycontrolled glaucoma using laser scanning confocal microscopy (LSCM). Materials and Methods: Fifty-five glaucomatous patients were enrolled and divided into two groups: Group 1 (27 eyes), controlled with one medication; and group 2 (28 eyes), controlled with two medications. Seventeen patients with dry eye disease (DED) and 17 healthy individuals served as controls. Patients completed the Ocular Surface Disease Index (OSDI) questionnaire and underwent determination of tear film break-up time (BUT), corneal staining, and Schirmer test I. For the GCD assessment, 12 high-quality images were acquired from the upper conjunctival epithelium (superior nasal, superior central, and superior temporal sectors). Results: Overall, GCD was significantly reduced in both glaucoma groups and those with DED compared to healthy controls $(p<0.001)$, with values markedly lower in group 2 compared to group 1 $(p<0.05)$. GCD was not significantly different between those with DED and group 2. A significant negative correlation was found of GCD with OSDI and with BUT $(p<0.001 ; R=-0.795$ and $R=-0.756$, respectively). Conclusion: Glaucoma therapy leads to a marked reduction of GCs, especially in the associative regimens. Given the negative correlation with tear film function tests, GCD reduction may play a pivotal role in
\end{abstract}

This article is freely accessible online.

Correspondence to: Marco Ciancaglini, Eye Clinic, San Salvatore Hospital, 66100 L'Aquila, Italy. Tel: +39 3499778688, Fax: +39 0854216636,e-mail: marco.ciancaglini@cc.univaq.it

Key Words: In vivo laser scanning confocal microscopy, conjunctiva, goblet cells, open-angle glaucoma, glaucoma therapy, ocular surface. the pathophysiology of the glaucoma-related disease of the ocular surface.

Glaucoma is a cause of irreversible blindness worldwide (1) and characterized by optic neuropathy with progressive loss of retinal ganglion cells and their axons, resulting in a permanent loss of the visual field. Glaucoma is a multifactorial disease, in which the intraocular pressure (IOP) increases due to an impairment of outflow structures (2), is the main and only modifiable risk factor, while neuroprotective drugs can complement hypotensive therapies $(3,4)$. The goal of glaucoma treatment is to maintain patient visual function and quality of life by lowering the IOP with therapy.

Medical therapy is the first-line approach in glaucoma treatment. Guidelines recommend initiating treatment with single medication (5); if the initial therapy is not effective, the addition of a second drug should be considered, combining agents with different and synergistic mechanisms of action. In the advanced stage of disease, several drugs may be combined to obtain low IOP values and reduce as much as possible the rate of damage progression.

The long-term use of anti-glaucoma medications produces several alterations of ocular surface components, with the most prominent changes involving the cornea, limbus, and conjunctiva (6-9). The most common epithelial modifications of the conjunctiva are squamous metaplasia, cellular desquamation and keratinization, dendritic cell activation, conjunctiva-associated lymphoid tissue activation, involution, and goblet cell (GCs) loss (7, 10, 11). In humans, GCs play crucial tasks in the homeostasis of the ocular surface since they are considered the main source of ocular surface mucoproteins, which are essential to maintain tear film stability. Thus, the loss of GCs progressively leads to a decrease in mucin production and along with other alterations leads to the induction of iatrogenic ocular surface disease (OSD) (12). 
GCs are key markers in assessing the severity of therapyrelated epithelial modifications because their number rapidly decreases after exposure to inflammatory and toxic effects of medications, although they tend to significantly recover when the irritating stimuli are relieved (13).

Nowadays, the most reliable method for analyzing GCs in vivo is laser scanning confocal microscopy (LSCM), a noninvasive in vivo technology that evaluates the ocular surface tissues at the cellular level, providing a valuable method to identify and characterize GC morphology in different OSDs including glaucoma-related OSD (14-16). Nevertheless, to date, no study was specifically dedicated to determining the effects of the commercially available classes of glaucoma medications, and their regimens, on the density of conjunctival GCs in patients with glaucoma.

The aim of this study was to evaluate by means of LSCM the GC density (GCD) and the modification of tear film function clinical tests in glaucomatous patients treated with different medication regimes. In addition, a comparison between glaucomatous patients, patients with dry eyes and healthy individuals was performed.

\section{Materials and Methods}

This was a case-control, cross-sectional, non-interventional study. The study adhered to the tenets of the Declaration of Helsinki and the approval from our Institutional Review Board (4121/2016) was obtained. Written informed consent was obtained from all patients prior to enrolment, after explanation of the nature of the study and possible consequences. All the study participants were consecutively enrolled at the Eye Clinic of the University of L'Aquila. We enrolled 55 consecutive Caucasian patients with glaucoma [primary open angle glaucoma (OAG), pseudo-exfoliative or pigmentary glaucoma] controlled with medical therapy who were referred to our Glaucoma Centre for routine visits.

For glaucomatous patients, the inclusion criteria were the following: best-corrected visual acuity $\geq 8 / 10$, refractive error $\leq 3$ diopters, mean IOP at the time of diagnosis higher than $22 \mathrm{mmHg}$ and well controlled at enrolment $(\mathrm{IOP}<18 \mathrm{mmHg}$ : mean of three measurements taken during the day) with one or two medications; the therapy had to be the same in both eyes during the 12 months prior to enrolment; central corneal thickness ranging from 530 to $570 \mu \mathrm{m}$, visual field test [30-2 test, full-threshold; Humphrey field analyzer II 750 (Carl Zeiss Meditec Inc., Dublin, CA, USA)] showing at least three contiguous points on the total deviation probability plot at the less than $2 \%$ level, Glaucoma Hemifield Test "outside normal limits" and ophthalmoscopic signs of glaucomatous optic neuropathy, which had to be consistent with the visual field alterations. The exclusion criteria were: history of systemic diseases or therapies in the previous 12 months that could have modified the status of the ocular surface; topical therapy other than anti-glaucoma medications, ocular surface inflammatory disease, previous ocular surgery or laser treatments, ocular trauma, end-stage glaucoma, pregnancy, and contact lens use.

Seventeen age- and gender-matched healthy individuals and 17 patients with Sjogren syndrome-related dry eye disease [DED, defined using International Dry Eye Workshop criteria (17) were included as controls. Exclusion criteria for patients with DED were: non-Sjogren syndrome-related DED, autoimmune deficiency syndrome, sarcoidosis, diabetes mellitus, corneal dystrophy, use of contact lens, previous ocular surgery, glaucoma, topical therapy with steroids or nonsteroidal anti-inflammatory drugs. Exclusion criteria for healthy controls were: history of systemic or topical therapy, ocular or systemic diseases in the previous 12 months, pregnancy, and contact lens use.

In cases in which both eyes were eligible for the study, only one eye per patient was randomly selected (by a computer-generated randomization) for the statistical analysis.

All patients underwent a complete ophthalmic evaluation that included best corrected visual acuity, refraction, Goldmann applanation tonometry, slit-lamp biomicroscopy, central corneal thickness measurement (ultrasound pachimetry), gonioscopy, ophthalmoscopy, visual field testing with full-threshold 30-2 test. Clinical tests included an Ocular Surface Disease Index (OSDI) (18) questionnaire and tear film function tests.

The OSDI is a 12-item disease-specific quality-of-life questionnaire with three subscales (ocular discomfort, ocular symptoms during daily activities, and environmental triggers) that assesses the impact of DED on vision-related quality of life. The frequency of ocular symptoms in the previous week was reported. A score from 0, indicating "none of the time," to 4, indicating "all of the time." was recorded, averaged and transformed to a scale ranging from 0 to 100 , with higher scores representing greater disabilities. Tear film function tests included, consecutively, breakup time (BUT), corneal staining, and Schirmer test I (STI, performed 30 minutes after BUT measurements), according to the Dry Eye WorkShop guidelines (19). BUT was recorded as the average of three consecutive measurements; STI results were expressed as the length of the strip that was wet after 5 minutes; corneal staining was evaluated with $1 \%$ sodium fluorescein (using the van Bijsterveld method) (20).

LSCM was performed using HRT III Rostock Cornea Module (RCM; Heidelberg Engineering GmbH, Dossenheim, Germany) 24 hours after clinical assessment in order to avoid artefacts due to the examinations of tear film, the use of topical anesthetics and sodium fluorescein. LSCM incorporates laser scanning optics with a detachable objective system for imaging the ocular surface and adnexa. The laser light with wavelength of $670 \mathrm{~nm}$, generates highcontrast and high-quality images with an area of $400 \times 400 \mu \mathrm{m}$, transversal optical resolution of $2 \mu \mathrm{m}$ and longitudinal optical resolution of $4 \mu \mathrm{m}$. Numerous types of information (both quantitative and qualitative) can be obtained when the system is coupled with image analysis software such as RCM (RCM cell count plugin for Image-J software; http://rsb.info.nih.gov/ij/ download.html). The microscope can be manually advanced through the full thickness of the tissue providing different optical section of the ocular tissue, but automated control of the optical section is only possible through a depth of $80 \mu \mathrm{m}$.

For GC assessment, sequential images derived from automatic scans and manual frame acquisition throughout upper bulbar conjunctiva with the eye in downward gaze (15-30 $\mu \mathrm{m}$ of depth) were acquired. Twelve high-quality images, without motion blur or compression lines, were selected from the three conjunctival sectors (superior nasal, superior central, superior temporal) to calculate the number of GCs using Cell Count Software (Heidelberg Engineering $\mathrm{GmbH})$ in manual mode. This definition was adopted taking into account reference images and the morphological characterization, 
Table I. Demographics and clinical data for the study subjects. Group 1: Patients with glaucoma controlled with one medication; group 2: patients with glaucoma controlled with two medications.

\begin{tabular}{lccccc}
\hline Group & $\begin{array}{c}\text { Age (years), } \\
\text { mean } \pm \text { SD }\end{array}$ & $\begin{array}{c}\text { Males/females, } \\
\mathrm{n}\end{array}$ & $\begin{array}{c}\text { IOP (mmHg), } \\
\text { mean } \pm \text { SD }\end{array}$ & $\begin{array}{c}\text { MD (db), } \\
\text { mean } \pm \text { SD }\end{array}$ & $\begin{array}{c}\text { Therapy (months), } \\
\text { mean } \pm \text { SD }\end{array}$ \\
\hline Control & $53.45 \pm 4.34$ & $8 / 9$ & $16.13 \pm 2.32$ & $+1.38 \pm 0.42$ & NA \\
DED & $54.89 \pm 5.67$ & $9 / 8$ & $15.74 \pm 3.76$ & $+1.54 \pm 0.36$ & $48 \pm 3.4$ \\
Group 1 & $56.12 \pm 3.92$ & $12 / 15$ & $16.28 \pm 2.62$ & $-2,67 \pm 1.18^{*}$ & $52 \pm 3.2$ \\
Group 2 & $57.48 \pm 2.12$ & $14 / 14$ & $16.96 \pm 1.57$ & $-4.38 \pm 2.26^{*}$ & $54 \pm 6.1$ \\
\hline
\end{tabular}

DED, Dry eye disease; MD, mean defect; NA, not applicable; SD, standard deviation. ${ }^{*} p<0.05$ versus healthy controls and DED.

as previously reported in the literature (21-23). The total confocal examination session lasted $10 \mathrm{~min}$ and none of the patients experienced particular complications at the end of the session.

A single experienced confocal operator (SDS) performed examinations and selected the images, which were evaluated by a second confocal operator (EB). The operators were masked for patient history and grouping during the image selection. After imaging, four randomly selected non-overlapping high-quality images of the nasal, central, and temporal sectors of the conjunctiva for each participant were considered for the analysis. GCD was the primary outcome measure; OSDI score, BUT, STI and fluorescein corneal staining score were secondary outcomes.

Statistical analysis. the variables were summarized as the mean and standard deviation (SD). Analysis was performed using SPSS ${ }^{\circledR}$ Advanced Statistical ${ }^{\mathrm{TM}}$ 13.0 Software (SPSS, Chicago, IL, USA). Student's $t$ - and Chi-squared test were used to evaluate age and gender differences, respectively, among healthy and glaucomatous patients. Mann-Whitney $U$-test was used to determine differences among groups of participants. Spearman's correlation coefficient (R) was performed to evaluate the relations between GCD and OSDI score, BUT, STI and corneal staining. Differences with $p$-values less than 0.05 were considered statistically significant.

\section{Results}

A total of 89 Caucasian patients were consecutively enrolled in this study, from January 2017 throughout May 2017. Table I summarizes the demographic and clinical data of all enrolled participants. Fifty-five eyes of 55 patients affected with medically controlled OAG were divided into two groups according to the number of medications: Group 1 (27 eyes) controlled with single therapy and Group 2 (28) eyes) receiving two drugs. Table II shows the therapy of glaucomatous patients at enrollment; medications and therapy regimens were not modified from treatment onset.

Seventeen eyes of age- and sex-matched patients with Sjogren syndrome-related DED who also were referred to our Ocular Surface Diseases Centre were enrolled.

A control group of seventeen eyes of 17 healthy patients (husbands and wives of patients not included in the study, who underwent examinations at the Eye Clinic of the University of L'Aquila) was also enrolled as controls.
Table II. Glaucoma medications in patients with glaucoma controlled with one medication (group 1) and in those controlled with two medications (group 2).

\begin{tabular}{llc}
\hline Groups & Therapy regimen & $\mathrm{N}$ \\
\hline Group 1 & Monotherapy & 27 \\
& Prostaglandin analogs & 10 \\
& Beta-blockers & 8 \\
& Bimatoprost 0.001\% & 4 \\
& 0.015\% Preservative-free tafluprost & 5 \\
Group 2 & Multi-therapy & 28 \\
& 0.005\% Latanoprost -0.5\% timolol fixed combination & 11 \\
& 0.004\% Travoprost -0.5\% timolol fixed combination & 4 \\
& 0.001\% Bimatoprost-0.5\% timolol fixed combination & 6 \\
& 0.001\% Bimatoprost and 0.5\% timolol, & \\
& unfixed combination & 2 \\
& Dorzolamide-0.5\% timolol fixed combination & 5 \\
\hline
\end{tabular}

Table III. Ocular surface clinical test for the study subjects. Group 1: Patients with glaucoma controlled with one medication; group 2: patients with glaucoma controlled with two medications.

\begin{tabular}{|c|c|c|c|c|}
\hline Group & $\begin{array}{c}\text { OSDI } \\
(\text { mean } \pm S D)\end{array}$ & $\begin{array}{c}\text { BUT } \\
(\text { mean } \pm S D)\end{array}$ & $\begin{array}{c}\text { STI } \\
(\text { mean } \pm \text { SD })\end{array}$ & $\begin{array}{l}\text { Corneal staining } \\
\quad(\text { mean } \pm \mathrm{SD})\end{array}$ \\
\hline Control & $8 \pm 0.3^{*}$ & $14 \pm 1.8^{*}$ & $15.1 \pm 2.5^{*}$ & $0.8 \pm 1.3 *$ \\
\hline DED & $49 \pm .6 .5^{\ddagger}$ & $5.3 \pm 1.7 \ddagger$ & $5.4 \pm 1.6^{\ddagger}$ & $3.5 \pm 1.6^{\ddagger}$ \\
\hline Group 1 & $25 \pm 4.2^{\dagger}$ & $10.3 \pm 2.3^{\dagger}$ & $10.8 \pm 2.1^{\dagger}$ & $1.8 \pm 1.1^{\dagger}$ \\
\hline Group 2 & $47 \pm 3.1$ & $7.6 \pm 2.1$ & $3.1 \pm 1.8$ & \\
\hline
\end{tabular}

BUT, Break-up time; DED, dry eye disease; OSDI, ocular surface disease index score; STI, Schirmer test I. ${ }^{*} p<0.001$ versus DED, group 1 and group 2 overall. ${ }^{\dagger} p<0.05$ versus group $2 .{ }^{\ddagger} p<0.05$ versus group 1 .

All ocular surface clinical test parameters (Table III) were significantly worse in the two glaucoma groups and those with DED compared to healthy controls $(p<0.001)$. Patients with DED had the worst clinical test values, whereas no 

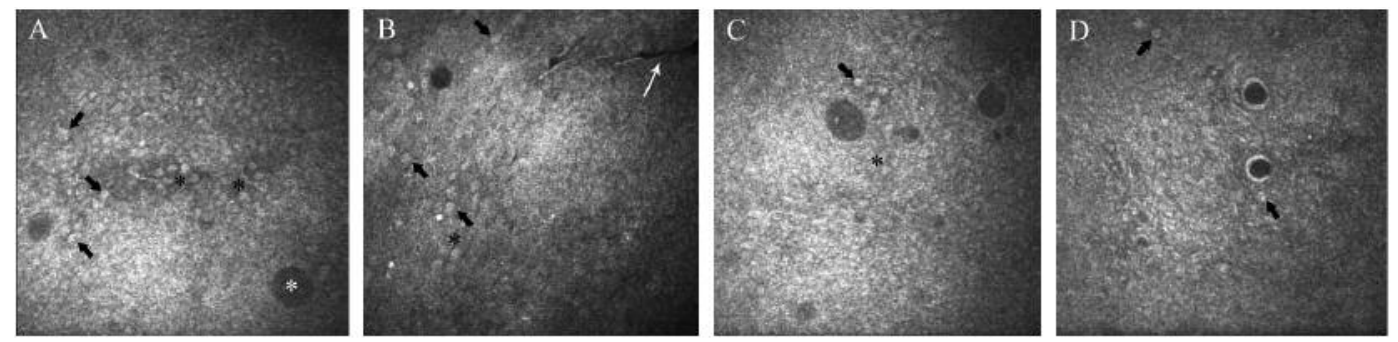

Figure 1. Laser confocal microscopy of conjunctival goblet cells (GCs). A: Healthy individual. GCs (black arrows) appear as hyper-reflective, round-shaped cellular elements dispersed within the epithelial cells, frequently exhibiting a clustering tendency (black asterisks). White asterisk indicates an intra-epithelial microcyst. B, C: Distribution of GCs in patients with glaucoma controlled with a single (B) and with two medications (C). White arrow represents a conjunctival fold. GC density appears markedly reduced in the patient treated with two compared to one medication. D: GC distribution in a patient with a Sjogren syndrome-related dry eye disease, where GCs are scattered and rare.

significant differences were found between those with DED and glaucoma treated with multi-therapy (group 2). Patients controlled with one drug (group 1) had significantly lower OSDI and corneal staining scores, and higher BUT and STI values compared to patients of group $2(p<0.05)$.

GCs were recognized in all enrolled participants and their morphological features were consistent with those reported in other confocal studies (21-23). They appeared as large cellular elements, hyper-reflective and oval-shaped, containing hyporeflective nuclei; their dimensions were greater than those of the surrounding epithelial cells, occasionally crowded in groups or dispersed within the epithelial layer of the conjunctiva, at 20-30 $\mu \mathrm{m}$ depth (Figure 1).

Overall, GCD was significantly reduced in both glaucoma groups and those with DED, compared to healthy controls $(p<0.001)$, with values markedly lower in group 2 compared to group $1(p<0.05)$. Moreover, a non-statistically significant difference was found between those with DED and those with glaucoma of group 2. The GCD of the study groups are described in Table IV. Correlation analysis between GCD and clinical data revealed a significant negative correlation between GCD and BUT $(p<0.001, \mathrm{R}=-0.756)$ and between GCD and OSDI $(p<0.01, \mathrm{R}=-0.795)$; conversely, ST1 and corneal staining did not show any significant correlation with GCD.

\section{Discussion}

In the past two decades, the introduction of LSCM as a diagnostic tool significantly increased the ability to analyze the ocular surface in patients with glaucoma. This technology is capable of showing several modifications of the ocular surface and adnexa at the cellular level, in vivo, permitting an imaging biopsy. In patients with glaucoma, LSCM was used to assess the modifications of the unconventional aqueous humor pathways as well as the alterations induced by anti-glaucoma drugs $(7,24,25)$.
Table IV. Conjunctival goblet cell density (GCD) for the study subjects. Group 1: Patients with glaucoma controlled with one medication; group 2: patients with glaucoma controlled with two medications.

\begin{tabular}{lcc}
\hline Group & GCD $\left(\right.$ cells $\left./ \mathrm{mm}^{2}\right)$, mean \pm SD & $p$-Value \\
\hline Control & $252.45 \pm 23.34$ & \\
DED & $117.63 \pm 15.38$ & $<0.001 \mathrm{vs}$. Control \\
Group 1 & $152.59 \pm 17.23$ & $<0.001 \mathrm{vs}$. Control \\
& & $<0.01 \mathrm{vs}$. DED \\
Group 2 & $119.21 \pm 17.10$ & $<0.001 \mathrm{vs}$. Control \\
& & $0.87 \mathrm{vs}$. DED \\
& & $<0.05 \mathrm{vs}$. Group 1 \\
\hline
\end{tabular}

DED, Dry eye disease.

In this LSCM study, we investigated the microscopic modifications of a specific cellular population of the conjunctiva, namely GCs, in patients with medically controlled glaucoma and in patients with DED. Overall, in both glaucoma and DED, we found a marked reduction in GCD and tear film function worsening in glaucoma, compared to healthy controls. In addition, the GC loss was significantly greater in patients treated with two drugs compared to those under monotherapy, without differences between glaucoma under multi-therapy and DED.

Since GCs work to maintain tear film stability on the ocular surface, their density may be considered a key factor in assessing the ocular surface status. GC loss has a significant clinical impact since it leads to dry eye, and dramatically reduces the clearance of toxic and inflammatory agents; in this way cytotoxic mediators persist on the ocular surface finally inducing chronic conjunctival inflammation $(6,26)$.

Several studies showed that GCs and mucin markers were reduced after long-term therapy with IOP lowering drugs whether containing preservative or preservative-free (PF) 
$(27,28)$. In a study using LSCM and impression cytology, Ciancaglini et al. showed a significant decrease of GCs after 6 months of therapy with benzalkonium chloride (BAK)preserved or PF levobunolol hydrochloride (61\% and $16 \%$, respectively) (29). In a different impression cytology study, Russ et al. found a transient increase in GCD in patients treated with preservative-containing prostaglandin analogs (PGAs), followed by a density reduction after longer periods of treatment (30). In line with this evidence, a further LSCM study observed a GCD increase after 1 month of therapy with BAK-preserved latanoprost, which was reduced to baseline values after 6 months; conversely, the use of PFtafluprost induced a short-term favorable stimulatory effect of on GCs, whose density remained stable at the 6-month follow-up (21). These results suggested that PF-PGAs may have a potentially positive effect on GCs compared to preservative-containing PGAs, in which the balance between the toxicity of preservative and stimulation by PGAs induces a long-term loss of GCs.

In the present study, we found higher GCD values for group 1 compared to group 2, those with DED and healthy controls. Since we did not sub-group patients belonging to of group 1 according to the class of medication and the presence of preservative (because of the limited sample size), we cannot state whether the higher GCD values are related to a higher percentage of patients taking PGAs or PF medications, or because of the reduced number of eye drop administrations during the day.

To date, no previous study has specifically investigated GCD in patients with glaucoma controlled with different therapeutic regimens. In fact, besides the previously mentioned studies conducted on monotherapy regimens (29, 30 ), only two recent LSCM studies investigated GCD values in multi-treated ( $\geq 3$ medications) patients with uncontrolled glaucoma scheduled to undergo filtration surgery. In these patients, GCD was found to be markedly reduced compared to that in healthy controls, with values two times lower than those found in the present study in patients taking two medications $(31,32)$. Even considering inter-individual variability, the lower GCD values documented in patients with uncontrolled glaucoma requiring surgery may be explained by the higher number of medications $(\geq 3 \mathrm{vs}$. 2 medications) and daily instillations, the higher daily cumulative BAK dosage, and a hypothetically more intensive treatment compared to patients with controlled glaucoma.

LSCM cannot determine whether the preservative or the active compound played the main role in the final GCD decrease because the present study did not consider this. Nevertheless, based on literature findings, one may state that the duration of therapy, the number of daily instillations, and the cumulative dose of preservative represent the main factors contributing to the GCD alterations in patients with glaucoma. Because of this, patients requiring an associative therapy, who represent a great part of the glaucoma population, are at higher risk of developing GCD alterations. These results were confirmed in the present study, in which patients receiving two drugs showed a greater GC loss compared to patients receiving a single medication. Interestingly, in patients receiving multi-treatment, the decrease of GCs was similar to that in patients with DED, suggesting that glaucoma medications and DED may lead to a similar effect in terms of conjunctival inflammation and risk of OSD. GCD was also significantly well correlated with both OSDI and BUT, which are the most commonly used clinical indicators of DED and OSD. Therefore, in patients with glaucoma, LSCM may be considered a valuable non-invasive procedure and finding of in vivo GCD decrease as a surrogate marker of ocular discomfort and worsening of quality of life.

Recently, pre-operative GCD and positivity for MUC5AC (the most important GC-derived mucin) were found to negatively correlate with the outcome of trabeculectomy (31, 32), a filtration surgical procedure in which an intra-scleral fistula drains the aqueous humor from the anterior chamber into the subconjunctival space (filtration bleb). In fact, patients who underwent surgery with high preoperative GCD values had a greater probability of having a functioning filtration bleb compared to patients undergoing surgery with a severe GC loss.

As well as ocular surface homeostasis, GCs have been reported to play an active role in the drainage of the aqueous humor through the bleb wall $(31,33)$. In fact, in an immunocytological and LSCM study, Amar et al. observed a great number of modified and atypical GCs on the conjunctival surface of functioning filtration blebs after trabeculectomy (33). At LSCM, they appeared as hyporeflective structures sometimes containing several nuclei, closely located to epithelial microcysts (EM), which have been considered as in vivo indicators of the trans-bleb aqueous humor passage (24). Three-dimensional spatial reconstruction of LSCM images further clarified the nature of these structures (34), showing that EM are close to but separated from the epithelium, and confirmed their involvement in the outflow of aqueous humor through the bleb wall after filtration surgery. All these studies supported the theory that modified GCs are active cytological carriers of the aqueous humor. Notably, EM were also found in untreated ocular hypertension (35), in patients with medically controlled primary open angle and low-tension glaucoma (36), in patients taking PGAs (37), and in patients with refractory glaucoma after ultrasonic cyclo-coagulation (38). This latter evidence further suggests that EM and, therefore, GCs are in vivo markers of unconventional outflow of aqueous humor in glaucomatous patients.

All this evidence strongly suggests that the preservation of GCs represents a crucial task in constraining OSD in 
patients controlled with medical therapy, and in increasing the likelihood of bleb survival in patients with uncontrolled glaucoma undergoing filtration surgery.

This study has certain limitation. Firstly, we included only white Caucasian patients, which did not allow evaluation of the racial differences in the GC population. Secondly, we enrolled patients with a similar duration of disease in different therapy groups: therefore, because baseline GC value before the initiation of therapy was unavailable, definitive conclusions about the progressive effects of therapy over time cannot be drawn. Further prospective studies are mandatory to answer this question. Thirdly, the presence of other conjunctival cell phenotypes with features similar to those of GCs, and a high inter-individual variation may introduce a bias in measuring GCD. Nevertheless, the GC features observed in our study were very similar to those reported in all other previous LSCM studies. Finally, we did not consider patients controlled with three or more medications since the data of ocular surface modifications with multi-therapies were previously reported $(31,32)$ and demonstrated a more severe toxic effect.

In conclusion, the present study confirmed that LSCM is a reliable method for the in vivo analysis of conjunctival GCs in patients with glaucoma, and that the density of these cells is objectively measurable and in line with previous literature findings. Anti-glaucoma medications have a detrimental effect on GCs, especially when an associative therapy is required to lower the IOP to the target value, leading to a condition quite similar to a iatrogenic DED. Given the role GCs play in the maintenance of ocular surface homeostasis, supported by the strong correlation between GCD and OSDI, these results should be carefully considered in order to limit and manage OSD.

\section{References}

1 Quigley HA and Broman AT: The number of people with glaucoma worldwide in 2010 and 2020. Br J Ophthalmol 90: 262-267, 2006.

2 Virno M, Sampaolesi R, Pecori Giraldi J, De Gregorio F, Taloni M, Brusini P, Di Staso S and Stecchi G: Ibopamine: D1dopaminergic agonist in the diagnosis of glaucoma. J Glaucoma 22: 5-9, 2013

3 Sena DF and Lindsley K: Neuroprotection for treatment of glaucoma in adults. Cochrane Database Syst Rev 25: 1, 2013.

4 Chiosi F, Keppel Hesselink J, Rinaldi M, Di Staso S, Bartollino S and Costagliola $\mathrm{C}$ : Phenytoin: its potential as neuroprotective and retinoprotective drug. Br J Clin Pharmacol 84(1): 195-196, 2017.

5 European Glaucoma Society Terminology and Guidelines for Glaucoma, 4th Edition - Chapter 3: Treatment principles and options Supported by the EGS Foundation. Br J Ophthalmol 101: 130-195, 2017.

6 Baudouin C, Labbè A and Liang H: Pauly A and BrignoleBaudouin F: Preservatives in eyedrops: the good, the bad and the ugly. Prog Retin Eye Res 29: 312-334, 2010.
7 Mastropasqua L, Agnifili L, Mastropasqua R and Fasanella V: Conjunctival modifications induced by medical and surgical therapies in patients with glaucoma. Curr Opin Pharmacol 13: 56-64, 2013.

8 Mastropasqua R, Agnifili L, Fasanella V, Curcio C, Brescia L, Lanzini M, Fresina M, Mastropasqua L and Marchini G: Corneoscleral limbus in glaucoma patients: In vivo confocal microscopy and immunocytological study. Invest Ophthalmol Vis Sci 56: 2050-2058, 2015.

9 Mastropasqua R, Agnifili L, Fasanella V, Lappa A, Brescia L, Lanzini M, Oddone F, Perri P and Mastropasqua L: In Vivo distribution of corneal epithelial dendritic cells in patients with glaucoma. Invest Ophthalmol Vis Sci 57: 5996-6002, 2016.

10 Agnifili L, Mastropasqua R, Fasanella V, Di Staso S, Mastropasqua A, Brescia L and Mastropasqua L: In vivo confocal microscopy of conjunctiva-associated lymphoid tissue in healthy humans. Invest Ophthalmol Vis Sci 55: 5254-5262, 2014.

11 Mastropasqua R, Agnifili L, Fasanella V, Nubile M, Gnama AA, Falconio G, Perri P, Di Staso S and Mariotti C: The conjunctivaassociated lymphoid tissue in chronic ocular surface diseases. Microsc Microanal 23: 697-707, 2017.

12 Doughty MJ and Bergmanson JPG: New insights into surface cells and glands of the conjunctiva and their relevance to the tear film. Optometry 74: 485-500, 2003.

13 Aragona P, Papa V, Micali A, Santocono M and Milazzo G: Long-term treatment with sodium hyaluronate-containing artificial tears reduces ocular surface damage in patients with dry eye. Br J Ophthalmol 86: 181-184, 2002.

14 Kobayashi A, Yoshita T and Sugiyama K: In vivo laser and white light confocal microscopic findings of the human conjunctiva. Ophthalmic Surg Lasers Imaging 35: 482-484, 2004.

15 Messmer EM, Mackert MJ, Zapp DM and Kampik A: In vivo confocal microscopy of normal conjunctiva and conjunctivitis. Cornea 25: 781-788, 2006.

16 Hong J, Zhu W, Zhuang H, Xu J, Sun X, Le Q, Li G and Wang $\mathrm{Y}$ : In vivo confocal microscopy of conjunctival goblet cells in patients with Sjogren's syndrome dry eye. Br J Ophthalmol 94: 1454-1458, 2010.

17 Lemp MA, Baudouin $\mathrm{C}$ and Baum $\mathrm{J}$ : The definition and classification of dry eye disease: report of the Definition and Classification subcommittee of the International Dry Eye WorkShop. Ocul Surf 5: 75-92, 2007.

18 Schiffman RM, Christianson MD, Jacobsen G, Hirsch JD and Reis BL: Reliability and validity of the ocular surface disease index. Arch Ophthalmol 118: 615-621, 2000.

19 Bron AJ, Abelson MB and Ousler G: Methodologies to diagnose and monitor dry eye disease: report of the Diagnostic Methodology subcommittee of the International Dry Eye WorkShop. Ocul Surf 5: 108-152, 2007.

20 Van Bijsterveld OP: Diagnostic tests in the sicca syndrome. Arch Ophthalmol 82: 10-14, 1969.

21 Mastropasqua L, Agnifili L, Fasanella V, Curcio C, Ciabattoni C, Mastropasqua R, Toto L and Ciancaglini M: Conjunctival goblet cells density and preservative-free tafluprost therapy for glaucoma: An in vivo confocal microscopy and impression cytology study. Acta Ophthalmol 91: e397-e405, 2013.

22 Mastropasqua L, Agnifili L, Mastropasqua R, Fasanella V, Nubile M, Toto L, Carpineto P and Ciancaglini M: In vivo laser scanning confocal microscopy of the ocular surface in glaucoma. Microsc Microanal 20: 879-894, 2014. 
23 Villani E, Sacchi M, Magnani F, Nicodemo A, Williams SE, Rossi A, Ratiglia R, De Cillà S and Nucci P: The ocular surface in medically controlled glaucoma: an in vivo confocal study. Invest Ophthalmol Vis Sci 57: 1003-1010, 2016.

24 Ciancaglini M, Carpineto P, Agnifili L, Nubile M, Fasanella V, Mattei PA, Mastropasqua L. Conjunctival characteristics in primary open-angle glaucoma and modifications induced by trabeculectomy with mitomycin $\mathrm{C}$ : an in vivo confocal microscopy study. Br J Ophthalmol 93: 1204-1209, 2009.

25 Agnifili L, Fasanella V, Costagliola C, Ciabattoni C, Mastropasqua R, Frezzotti $\mathrm{P}$ and Mastropasqua L: In vivo confocal microscopy of meibomian glands in glaucoma. Br J Ophthalmol 97: 343-49, 2013.

26 Pisella PJ, Debbasch C and Hamard P: Conjunctival proinflammatory and proapoptotic effects of latanoprost and preserved and unpreserved timolol: an ex vivo and in vitro study. Invest Ophthalmol Vis Sci 45: 1360-1368, 2004.

27 Liang H, Baudouin C, Pauly A and Brignole-Baudouin F: Conjunctival and corneal reactions in rabbits following shortand repeated exposure to preservative-free tafluprost, commercially available latanoprost and $0.02 \%$ benzalkonium chloride. Br J Ophthalmol 92: 1275-1282, 2008.

28 Kahook MY and Noecker R: Quantitative analysis of conjunctival goblet cells after chronic application of topical drops. Adv Ther 25: 743-751, 2008.

29 Ciancaglini M, Carpineto P, Agnifili L, Nubile M, Fasanella V, Lanzini M, Calienno R and Mastropasqua L: An in vivo confocal microscopy and impression cytology analysis of preserved and unpreserved levobunolol-induced conjunctival changes. Eur J Ophthalmol 18: 400-407, 2008.

30 Russ HH, Costa VP and Ferreira FM: Conjunctival changes induced by prostaglandin analogues and timolol maleate: a histomorphometric study. Arq Bras Oftalmol 70: 910-916, 2007.

31 Agnifili L, Fasanella V and Mastropasqua R: In vivo goblet cell density as a potential indicator of glaucoma filtration surgery outcome. Invest Ophthalmol Vis Sci 57: 2898-2905, 2016.

32 Mastropasqua R, Fasanella V, Brescia L, Oddone F, Mariotti C, Di Staso $\mathrm{S}$ and Agnifili L: In vivo confocal imaging of the conjunctiva as a predictive tool for the glaucoma filtration surgery outcome. Invest Ophthalmol Vis Sci 58: 114-120, 2017.
33 Amar N, Labbe A, Hamard P, Dupas B and Baudouin C: Filtering blebs and aqueous pathway: an immunocytological and in vivo confocal microscopy study. Ophthalmology 115: 11541161,2008

34 Di Staso S, Agnifili L, Di Gregorio A, Climastone H, Galassi E, Fasanella V and Ciancaglini M: Three-dimensional laser scanning confocal analysis of conjunctival microcysts in glaucomatous patients before and after trabeculectomy. In Vivo 31: 1081-1088, 2017.

35 Ciancaglini M, Carpineto P, Agnifili L, Nubile M, Fasanella V and Mastropasqua L: Conjunctival modifications in ocular hypertension and primary open angle glaucoma: An in vivo confocal microscopy study. Invest Ophthalmol Vis Sci 49: 30423048,2008 .

36 Agnifili L, Carpineto P, Fasanella V, Mastropasqua R, Zappacosta A, Di Staso S, Costagliola C and Mastropasqua L: Conjunctival findings in hyperbaric and low-tension glaucoma: An in vivo confocal microscopy study. Acta Ophthalmol 90: e132-e137, 2012.

37 Mastropasqua R, Fasanella V, Pedrotti E, Lanzini M, Di Staso $\mathrm{S}$, Mastropasqua L and Agnifili L: Trans-conjunctival aqueous humor outflow in glaucomatous patients treated with prostaglandin analogues: an in vivo confocal microscopy study. Graefes Arch Clin Exp Ophthalmol 252: 1469-1476, 2014.

38 Mastropasqua R, Agnifili L, Fasanella V, Toto L, Brescia L, Di Staso S, Doronzo E and Marchini G: Uveo-scleral outflow pathways after ultrasonic cyclocoagulation in refractory glaucoma: an anterior segment optical coherence tomography and in vivo confocal study. Br J Ophthalmol 100: 1668-1675, 2016.

Received December 18, 2017

Revised January 24, 2018

Accepted January 29, 2018 